\title{
Interactive comment on "Investigation of several proxies to estimate sulfuric acid concentration in volcanic plume conditions" by Clémence Rose et al.
}

\section{Anonymous Referee \#3}

Received and published: 9 October 2020

General Comments: This manuscript presents a comparison of H2SO4 proxies used to estimate sulfuric acid in a volcanic plume. While there are various proxies in the literature to estimate $\mathrm{H} 2 \mathrm{SO} 4$ (a difficult species to measure) their use in environments with large SO2 and thus $\mathrm{H} 2 \mathrm{SO} 4$ concentrations has not been explored. To this end, using previously published approaches, the authors developed 8 different proxies for estimating $\mathrm{H} 2 \mathrm{SO} 4$ concentrations using various parameterizations SO2 concentrations, solar radiation, condensation sink and relative humidity. These proxies were then compared against $\mathrm{H} 2 \mathrm{SO} 4$ measurements of the volcanic plume from the Maïdo observatory located on La Réunion Island. Results showed that the proxies where the weights of the individual parameters were adjusted gave the best agreement compared to mea- 
surements. A proxy using an additional sink term related to cluster formation gave good agreement over the whole range of measured $\mathrm{H} 2 \mathrm{SO} 4$ concentrations. These proxies were also compared to airborne measurements of $\mathrm{H} 2 \mathrm{SO} 4$ concentrations of the volcanic plume from Mt. Etna. Here the best proxy-measurement agreement was observed when the dependence of predicted $\mathrm{H} 2 \mathrm{SO} 4$ concentration to CS was the lowest, and when the dependence on $\mathrm{SO} 2$ concentration was simultaneously the highest. Comparison of an existing literature proxy with the two data sets also gave reasonable agreement in these high $\mathrm{H} 2 \mathrm{SO} 4$ concentration environments, yet not to the level of the newly developed proxies.

In all I found this paper to be well thought out and written. One aspect I found to be missing was a discussion of the uncertainties in both the proxies and the measurements. $\mathrm{H} 2 \mathrm{SO} 4$ measurements in general have associated uncertainties on the order of $+/-35-45 \%$, however in this work none were reported. The parameters used in the proxy calculations also have associated uncertainties resulting in an overall uncertainty in the estimated H2SO4 concentration. These uncertainties (both the measurements and calculations) need to be discussed in terms of the comparisons.

Also at such high $\mathrm{H} 2 \mathrm{SO} 4$ concentrations (approaching 10e9 molecule cm-3), the reagent ion will be depleted in the NO3 CIMS measurements. The stated concentration calculation assumes pseudo first order kinetics which most likely are no longer applicable under these conditions. Were calibrations performed using these large $\mathrm{H} 2 \mathrm{SO} 4$ concentrations to prove the validity of the calculation?

Another aspect was the discussion of the airborne measurements. The best agreement between proxy and measurements was observed when no CS term was included (not very realistic). It was also stated that there were gaps in radiation data caused by improper measurements during turns, when the aircraft itself affected the amount of radiation reaching the sensor. Was upwelling radiation considered in these calculations? Assuming these measurements were made inside the plume with particles present, the nadir or reflected radiation could approach that of the zenith or incoming.

Printer-friendly version

Discussion paper
Interactive

comment 
An increase in the $\mathrm{H} 2 \mathrm{SO} 4$ production term could balance the inclusion of the CS term.

I would find a statement about the estimated lifetime of gas phase $\mathrm{H} 2 \mathrm{SO} 4$ under these high $\mathrm{H} 2 \mathrm{SO} 4$ conditions useful too.

Below are some specific comments and suggested grammatical changes. One large one to mention here is the omission of the proxy equation (Eq. 11) from Mikkonen 2011. It is useful and relevant to this work and not proper to ask the reader to look up.

I feel that if these comments and suggestions are addressed, this work would be a fine contribution to ACP.

Specific Comments:

All Fig. $X$ should be Figure $X$ in text

Consider replacing "regular conditions" with "low SO2 conditions"

Page 6, line 19: with had a -> which had a

Page 6, line 27: in and off-plume -> in and out of plume

Lower median radiation during STRAP then OCTAVE due to diurnal observations during OCTAVE. Why not compare same time of day?

Page 7, line 1: in and off-plume -> in and out of plume (you use outside plume in line 3)

Figure 1 - Why not use black for radiation similar to other plots. Yellow for radiation is hard to see. Consider changing the color.

Page 10, lines 1-3: Equation is busy and unnecessary

Page 10, line 10: define 'k'

Printer-friendly version

Page 10, Line 11: we defined in parallel proxy A1 -> we defined a parallel proxy A1

Discussion paper

Page 10, line 18: CS was first removed in proxies $F 2$ and $A 2$, and it was re- 
introduced... -> the CS was first removed in proxies F2 and A2, and re-introduced...

Page 10, line 22: respectively for "F" and "A" proxies. -> respectively for the "F" and "A" ACPD proxies.

Page 10, line 24 : contribute up to $\sim 35 \%$ to $->$ contribute up to $\sim 35 \%$ of

Interactive

Page 10, lines 29-30: definitions of alpha and beta need to come earlier.

comment

Page 11, line 13: which is defined as follow for -> which is defined as follows for

Page 11 Table 2: parameters need to be defined, especially $\mathrm{k}$. Is $\mathrm{K} \times \mathrm{k}=\mathrm{k}$ ' in equation 6 ? As general rule the reader should not have to read the text to understand a figure or table.

Page 12, line 12: in a different volcanic plume, in which the conditions however overall resembled the average conditions encountered during OCTAVE. - $>$ in a different volcanic plume with conditions similar to those encountered during OCTAVE.

Page 12, line 13: and we believe that their behaviour was not well caught due to their limited number. -> and due to the limited number of measurements probably do not represent $\mathrm{H} 2 \mathrm{SO} 4$ concentrations under such large concentrations of $\mathrm{SO} 2$ as a whole.

Page 12, line 30: measurement -> measurements

Page 13, line 1: make them better -> improve results

Page 13, line 6: in regular conditions, who noticed a better performance of the proxies when taking $\mathrm{RH}$ into account. -> who noticed a better performance of the proxies when taking $\mathrm{RH}$ into account under regular conditions.

Page 13, line 22: point up that overall -> point out that the overall

Printer-friendly version

Page 13, line 23: shown on -> shown in

Page 14, line 23: Eq. (11). At first look I thought this referred to Eq. (11) in the present work, which doesn't exist. I'm assuming this refers to Eq. (11) in Mikkonen et 
al. (2011). The equation needs to be added here so the reader doesn't have to go look it up!

Page 19, line 5: CS -> CSs

Page 23, line 15: The condensation sink (CS) was in a first approach considered as the only sink contributing to the balance of [H2SO4] to derive seven proxies based on the knowledge of $\mathrm{SO} 2$ mixing ratios, global radiation, $\mathrm{CS}$ and $\mathrm{RH}$, which inclusion in the sink term was tested in several formulations. -> The condensation sink (CS) was in a first approach considered as the only sink contributing to the balance of [H2SO4] to derive seven proxies based on the knowledge of $\mathrm{SO} 2$ mixing ratios, global radiation, and $\mathrm{CS}$. $\mathrm{RH}$, included in the sink term was tested in several formulations.

Page 23, line 28: In contrast, the inclusion of $\mathrm{RH}, \ldots$ This sentence is long and runs on. Needs to be rewritten and split up to convey conclusions.

Page 24, line 1: and on average higher at - but was on average higher at

Page 24, line 4: the literature -> that work

Interactive comment on Atmos. Chem. Phys. Discuss., https://doi.org/10.5194/acp-2020-636, 2020. 